\title{
Qualidade do cuidado: Avaliação da disponibilidade de insumos, imunobiológicos e medicamentos na Atenção Básica em município de Minas Gerais, Brasil
}

\section{Quality of care: Evaluation of the availability of inputs, immunobiologicals and drugs in Primary Care in a municipality of Minas Gerais, Brazil \\ Calidad del cuidado: Evaluación de la disponibilidad de insumos, inmunobiológicos y medicamentos en la Atención Básica en el municipio de Minas Gerais, Brasil}

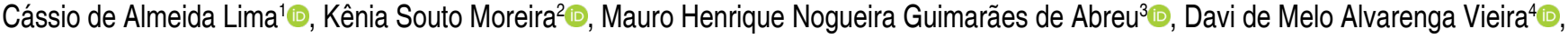 \\ Sabrina Aparecida de Lima Mangueira ${ }^{5}$, Maria Aparecida Vieira ${ }^{1}$, Simone de Melo Costa $^{1}(\mathbb{D})$ \\ ${ }^{1}$ Universidade Estadual de Montes Claros (UNIMONTES). Montes Claros, MG, Brasil. \\ ${ }^{2}$ Centro Universitário Pitágoras de Montes Claros (UNIFIPMoc). Montes Claros, MG, Brasil. \\ ${ }^{3}$ Universidade Federal de Minas Gerais (UFMG). Belo Horizonte, MG, Brasil. \\ ${ }^{4}$ Fundação Educacional Dom André Arcoverde, Centro de Ensino Superior de Valença (CESVA/FAA). Valença, RJ, Brasil. \\ ${ }^{5}$ Faculdades Santo Agostinho de Montes Claros (FASA). Montes Claros, MG, Brasil.
}

\section{Resumo}

Objetivo: Avaliar a Atenção Básica quanto aos insumos, imunobiológicos e medicamentos disponibilizados nos serviços de saúde da família de município de Minas Gerais, Brasil. Métodos: Trata-se de pesquisa transversal, analítica, conduzida em 2014, a partir de instrumento ministerial intitulado Autoavaliação para Melhoria do Acesso e da Qualidade da Atenção Básica, de equipes de saúde da família. Oito itens foram considerados, com valores entre zero a 10 pontos, da total inadequação à total adequação da Unidade Básica de Saúde (UBS), ao item avaliado. Classificaram-se as unidades em padrões de qualidade "muito insatisfatório" a "muito satisfatório" e analisou-se conforme localização rural e urbana, nível de significância $p<0,05$. Resultados: Participaram 75 equipes, que apresentaram expressiva inadequação nos itens: UBS dispor todas as vacinas exigidas no calendário básico, $5,95( \pm 4,003)$; possuir insumos e medicamentos para abordagem inicial de urgência/emergência, 2,33 $( \pm 2,500)$; apresentar os medicamentos básicos com regularidade, 3,68 $( \pm 3,146)$. A classificação foi regular para $45,3 \%$ das unidades e insatisfatória para $29,3 \%$, sem diferenças quanto à localização urbana ou rural $(p=0,479)$. Conclusão: Evidenciou-se avaliação insatisfatória, com inadequações relativas às vacinas, à abordagem inicial nas situações de urgência e emergência e à disponibilidade dos medicamentos. Tal situação prejudica o elenco de ações e a resolutividade dos serviços, afetando a capacidade da equipe para atender, efetivamente, as demandas de saúde da população.

Palavras-chave: Avaliação em Saúde; Pesquisa sobre Serviços de Saúde; Atenção Primária à Saúde; Estratégia Saúde da Família; Insumos Farmacêuticos

Como citar: Lima CA, Moreira KS, Abreu MHNG, Vieira DMA, Mangueira SAL, Vieira MA, et al Qualidade do cuidado: Avaliação da disponibilidade de insumos, imunobiológicos e medicamentos na Atenção Básica em município de Minas Gerais, Brasil. Rev Bras Med Fam Comunidade. 2019;14(41):1900. https://doi.org/10.5712/rbmfc14(41)1900

Autor correspondente: Cássio de Almeida Lima. E-mail: cassioenf2014@gmail.com Fonte de financiamento: Conselho Nacional de Desenvolvimento Científico e Tecnológico (CNPq), Processo 437324/2016-8.

Parecer CEP:

704.718 (UNIMONTES), aprovado em 01/07/2014.

Procedência e revisão por pares: revisado por pares.

Recebido em: 15/10/2018. Aprovado em: 26/02/2019. 


\begin{abstract}
Objective: To evaluate the Basic Care regarding the supplies, immunobiologicals and medicines available in the family health services of the municipality of Minas Gerais, Brazil. Methods: Cross-sectional, analytical research, conducted in 2014, from a ministerial instrument titled Self-Assessment for Improving Access and Quality of Primary Care, of family health teams. Eight items were considered, with values between zero and 10 points, of the total inadequacy to the total adequacy of the Basic Health Unit (UBS), to the evaluated item. The units were classified in quality standards "very unsatisfactory" to "very satisfactory" and analyzed according to rural and urban locations, level of significance $p<0.05$. Results: 75 teams participated, which presented significant inadequacy in the items: UBS dispose of all the vaccines required in the basic calendar, 5.95 ( \pm 4.003$)$; to have supplies and medicines for the initial emergency/emergency approach, $2.33( \pm 2.500)$; presenting the basic medicines regularly, $3.68( \pm 3.146)$. The classification was regular for $45.3 \%$ of the units and unsatisfactory to $29.3 \%$, without differences in urban or rural locations $(p=0.479)$. Conclusion: There was an unsatisfactory evaluation, with inadequacies related to vaccines, the initial approach in emergency and emergency situations and the availability of medicines. Such a situation undermines the list of actions and the resolution of services, affecting the capacity of the team to effectively meet the population's health demands.
\end{abstract}

Keywords: Health Evaluation; Health Services Research; Primary Health Care; Family Health Strategy; Pharmaceutical Raw Material

\title{
Resumen
}

Objetivo: Evaluar la Atención Básica en cuanto a los insumos, inmunobiológicos y medicamentos disponibles en los servicios de salud de la familia del municipio de Minas Gerais, Brasil. Métodos: Se trata de investigación transversal, analítica, conducida en 2014, a partir de instrumento ministerial titulado Autoevaluación para la mejora del acceso y de la calidad de la atención básica, de los equipos de salud de la familia. Se consideraron ocho ítems, con valores entre cero y diez puntos, de la total inadecuación a la total adecuación de la Unidad Básica de Salud (UBS), al ítem evaluado. Se clasificaron las unidades en estándares de calidad "muy insatisfactorio" a "muy satisfactorio" y se analizó según ubicación rural y urbana, nivel de significancia $p<0,05$. Resultados: Participaron 75 equipos, que presentaron expresiva inadecuación en los ítems: UBS disponer todas las vacunas requeridas en el calendario básico, $5,95( \pm 4,003)$; poseer insumos y medicamentos para el enfoque inicial de urgencia/emergencia, $2,33( \pm 2,500)$; presentar los medicamentos básicos con regularidad, 3,68 $( \pm 3,146)$. La clasificación fue regular para el $45,3 \%$ de las unidades e insatisfactoria para el $29,3 \%$, sin diferencias en cuanto a la ubicación urbana o rural $(p=0,479)$. Conclusión: Se evidenció una evaluación insatisfactoria, con inadecuaciones relativas a las vacunas, al abordaje inicial en las situaciones de urgencia y emergencia y a la disponibilidad de los medicamentos. Tal situación perjudica el elenco de acciones y la resolutividad de los servicios, afectando la capacidad del equipo para atender efectivamente las demandas de salud de la población.

Palabras clave: Evaluación en Salud; Investigación en Servicios de Salud; Atención Primaria de Salud; Estrategia de Salud Familiar; Insumos Farmacéuticos

\section{Introdução}

O Ministério da Saúde brasileiro propôs a Estratégia de Saúde da Família (ESF) como um novo modelo em Atenção Básica (AB). Após a sua implantação, a abrangência da ESF foi ampliada e incorporaram-se os preceitos da Política Nacional de Atenção Básica (PNAB), com os atributos de uma atenção integral. A ESF foi adotada como meio prioritário para reorientar a assistência no Sistema Único de Saúde (SUS). ${ }^{1-3}$ Todavia, a política de expansão da ESF, no país, não foi sistematicamente monitorada, ao longo do tempo. Ela foi acompanhada por avaliações esparsas. ${ }^{1}$

Nesse contexto, surgiram propostas de mudanças nas diretrizes da PNAB. E, a partir de 2012, o Ministério da Saúde brasileiro instituiu o Programa Nacional de Melhoria do Acesso e da Qualidade da Atenção Básica (PMAQ-AB). ${ }^{4}$ Trata-se de estratégia promotora de um processo permanente e progressivo para ampliar o acesso, bem como qualificar a gestão da $A B$ e o cuidado à saúde da comunidade. $A$ avaliação, pelo PMAQ-AB, permite reconhecer e analisar as formas como estão organizadas as ações no nível primário de atenção à saúde..$^{1,4,5}$

O PMAQ-AB almeja incentivar os gestores e as equipes de saúde da família a melhorar a qualidade dos serviços e da assistência ofertados aos usuários do território. Apresenta um conjunto de estratégias para qualificação, acompanhamento e avaliação do trabalho dessas equipes, incluindo também a análise 
das condições de estrutura e funcionamento das unidades de saúde. Em sua implantação, o programa é organizado em quatro fases, as quais compõem um ciclo e se complementam: adesão e contratualização, desenvolvimento (em que se insere a autoavaliação das equipes), avaliação externa e recontratualização. ${ }^{1,4}$

Ressalta-se que o referido Programa fornece dados multidimensionais com inúmeras possibilidades de análises. ${ }^{6}$ Entre as dimensões contempladas no PMAQ-AB, encontra-se a subdimensão que objetiva averiguar os fatores relacionados aos insumos, medicamentos, imunobiológicos e vacinas utilizados na assistência em saúde. ${ }^{4}$

Embora tenham se observado melhorias nessa subdimensão e sejam indubitáveis os avanços na $A B$ brasileira, ainda persistem consideráveis desafios a serem superados. ${ }^{2,3,7,8}$ Podem ser citados: a inadequação física das unidades de saúde; o financiamento limitado; a insuficiente integração da $A B$ com os serviços de saúde, a fim de garantir o cuidado contínuo e coordenado na rede de atenção; e a carência de recursos humanos com qualificação apropriada para o cuidado na $A B^{2,3,5-8} E$, para que a ESF realmente consolide como AB de qualidade, que possa ser resolutiva e coordenadora do cuidado, é premente enfrentar as dificuldades relativas à estrutura das unidades de saúde, em que se destacam a infraestrutura, os equipamentos, imunobiológicos e medicamentos. ${ }^{8}$

Sendo assim, os fatores desfavoráveis supracitados requerem maior atenção e intervenções dos gestores e profissionais de saúde para alcançar a máxima qualidade do cuidado ofertado na $E S F .{ }^{7-9} E$ o PMAQ-AB propicia uma melhor reflexão sobre o modelo de estruturação dos serviços, subsidiando o planejamento, assim como o monitoramento e a avaliação das unidades da AB. A análise das subdimensões desse Programa tem potencial para balizar as decisões e direcionar a PNAB, contribuindo, ainda, na perspectiva da atenção à saúde em uma dimensão integral e universal. ${ }^{1,10}$

O conhecimento oriundo da avaliação da ESF norteia o planejamento de ações que possam retificar as inadequações encontradas nas unidades de saúde e permitir a plena operacionalização da $A B$, pela visão dos aspectos facilitadores e dificultadores, que comprometem a qualidade da assistência. ${ }^{10}$

Este estudo objetivou avaliar a Atenção Básica quanto aos insumos, imunobiológicos e medicamentos disponibilizados nos serviços de saúde da família de município de Minas Gerais (MG), Brasil.

\section{Métodos}

Estudo de delineamento transversal, com análise descritiva e analítica. Foi realizado em Montes Claros, cidade polo, de médio porte, situada na região norte do estado de MG - Brasil. Conforme levantamento demográfico do Instituto Brasileiro de Geografia e Estatística (IBGE) de 2010, a população do município era de 361.915 habitantes. O Índice de Desenvolvimento Humano Municipal (IDH-M) médio é de 0,770 e o Índice de Gini 0,5391. O local é referência em setores de prestação de serviços, comércio, educação e saúde. ${ }^{11}$

Os serviços da ESF compõem a rede de $A B$ local desde 1992. A partir de então, o número de equipes e a cobertura populacional têm crescido progressivamente, consubstanciando o modelo de saúde da família como principal forma de organização da AB local, sendo que, hoje, esta cobre $100 \%$ da população. Existem os programas de Residência Médica em Medicina de Família e Comunidade e de Residência Multiprofissional em Saúde de Família, iniciados em 1999; e atualmente também os programas de Residência Multiprofissional em Saúde Mental e de Enfermagem Obstétrica. ${ }^{12}$ 
Este trabalho trata dos resultados da autoavaliação dos profissionais, que ocorreu durante o segundo ciclo do PMAQ-AB. Participaram da atual pesquisa todas as 75 equipes de saúde da família cadastradas em 2014 no município. Entre essas equipes, 64 prestavam serviços à população residente na área urbana (localização urbana) e 11 às comunidades rurais (localização rural).

Os dados da pesquisa, de caráter secundário, foram levantados a partir de formulários da Autoavaliação para Melhoria do Acesso e da Qualidade da Atenção Básica (AMAQ). O instrumento é composto por dimensões e subdimensões, que permitem a análise de maneira independente. ${ }^{4}$ Os formulários, preenchidos pelas equipes, foram obtidos em site do Ministério da Saúde - no endereço eletrônico http://www.saudedafamilia.net/questionário (não mais disponível para acesso), acesso nos dias 7 e 8 de maio de 2014, após autorização formal da Secretaria Municipal de Saúde.

Neste trabalho, considerou-se a subdimensão “Insumos, Imunobiológicos e Medicamentos”, integrante da dimensão "Unidade Básica de Saúde (UBS)", do citado instrumento da AMAQ. ${ }^{4}$ Essa subdimensão considera oito padrões de qualidade da UBS, descritos no Quadro 1 na íntegra.

Quadro 1. Descrição dos oito itens avaliados da subdimensão "Insumos, Imunobiológicos e Medicamentos", da Autoavaliação para Melhoria do Acesso e da Qualidade da Atenção Básica. Montes Claros, Minas Gerais, Brasil, 2014.

\section{A Unidade Básica de Saúde dispõe de: $:^{4: 42-44}$}

1) "Insumos em quantidade suficiente para o desenvolvimento regular das ações de saúde e atividades educativas".

2) "Materiais e insumos necessários para o trabalho dos Agentes Comunitários de Saúde".

3) "Todas as vacinas do calendário básico do Programa Nacional e Estadual de Imunização".

4) "Material impresso em quantidade suficiente para o desenvolvimento regular das ações em saúde".

5) "Insumos e medicamentos indicados para o primeiro atendimento nos casos de urgência e emergência".

6)“Equipamentos de proteção individual de forma regular e suficiente”.

7) "Medicamentos do Componente Básico da Assistência Farmacêutica com suficiência e regularidade".

8) "Mecanismos de monitoramento e controle de estoque (abastecimento) de materiais/insumos, medicamentos e imunobiológicos e das condições adequadas de conservação destes".

Fonte: instrumento da Autoavaliação para Melhoria do Acesso e da Qualidade da Atenção Básica (Brasil, 2012). 4:42-44

Cada padrão mensura a adequação dos insumos, imunobiológicos e medicamentos disponíveis nas UBS para as ações na AB, ou seja, avaliar o padrão de qualidade. Cada item é avaliado em escala de zero a dez pontos. A pontuação zero diz respeito ao não cumprimento do padrão, ou seja, total inadequação da unidade. $E$ dez equivale à total adequação. ${ }^{4} \mathrm{~A}$ partir da soma dos pontos, classifica-se a equipe em padrões de qualidade: i) muito insatisfatório (0-15 pontos); ii) insatisfatório (16-31); regular (32-47); iii) satisfatório (48-63); iv) muito satisfatório (64-80). A pontuação máxima na subdimensão estudada é de 80 pontos, isto é, dez pontos para cada item/questão. ${ }^{4} \mathrm{~A}$ análise das equipes quanto a tais pontuações e classificações se deu conforme as instruções contidas no instrumento da AMAQ, do PMAQ-AB. ${ }^{4}$

Adicionalmente, as equipes investigadas foram categorizadas em localização urbana ou rural. Isso ocorreu a partir de consulta dos pesquisadores no setor de gestão de dados da Coordenação de $A B$ da Secretaria Municipal de Saúde, efetuada no Sistema de Informação da Atenção Básica (SIAB).

O processamento estatístico dos dados foi efetuado no Software IBM SPSS 22.0. A descrição dos resultados foi apresentada em valores de médias, desvio padrão (DP), percentis 25, 50 e 75, para pontuação de cada item avaliado. Os números absolutos e percentuais de equipes classificadas nos 
diferentes padrões de qualidade foram descritos (muito insatisfatório a muito satisfatório). Para comparar as médias da pontuação das equipes em cada padrão mensurado na subdimensão, por localização (urbana e rural), utilizou-se o teste t Student, devido à normalidade na distribuição dos dados, certificada pelo teste Kolmogorov Smirnov ( $p=0,200)$.

A igualdade das variâncias foi verificada pelo Levene's Test. As variáveis 'Todas vacinas do calendário básico', 'Material impresso para ações em saúde', 'Medicamentos básicos com regularidade' e 'Mecanismos de monitoramento e controle de estoque e conservação adequada' não apresentaram igualdade de variância em ambos os grupos. Para essas variáveis, adotou-se o teste t Student para variância desigual. Ainda, associou-se a classificação nos padrão de qualidade das equipes conforme localização, seja urbana ou rural, pelo teste Qui-quadrado de tendência linear, Linear-by-linear association, em respeito à ordenação natural dos níveis de satisfação. O nível de significância adotado foi $p<0,05$.

O protocolo de pesquisa foi aprovado pelo Comitê de Ética em Pesquisa Envolvendo Seres Humanos da Universidade Estadual de Montes Claros (Unimontes), com Parecer Consubstanciado n 704.718/2014. Também se obteve concordância institucional da Secretaria Municipal de Saúde.

\section{Resultados}

O número total de pontos na subdimensão, por equipe, variou de 3 a 72 pontos, com média igual a $39,21( \pm 13,351)$ pontos. Constatou-se que $75 \%$ das equipes apresentaram até 47 pontos.

A Tabela 1 apresenta a descrição de oito itens de qualidade, avaliados pelas equipes vinculadas à saúde da família. Vale observar os seguintes resultados insatisfatórios nos itens (média e DP): UBS dispor todas as vacinas exigidas no calendário básico, $5,95( \pm 4,003)$; possuir insumos e medicamentos para atendimento inicial de urgência/emergência, 2,33 ( $\pm 2,500)$; e UBS apresenta os medicamentos básicos com regularidade, 3,68 $( \pm 3,146)$.

Tabela 1. Descrição dos valores médios e separatrizes da pontuação em cada padrão de qualidade para Insumos, Imunobiológicos e Medicamentos na Unidade Básica de Saúde (UBS). Equipes de saúde da família, Montes Claros, Minas Gerais, Brasil, 2014. $n=75$

\begin{tabular}{|c|c|c|c|c|c|}
\hline Padrão de qualidade (UBS dispõe de...) & Média & $\begin{array}{l}\text { Desvio } \\
\text { Padrão }\end{array}$ & $\begin{array}{c}\text { Percentil } \\
25\end{array}$ & $\begin{array}{c}\text { Percentil } \\
50\end{array}$ & $\begin{array}{c}\text { Percentil } \\
75\end{array}$ \\
\hline 1) Insumos para ações de saúde e atividades educativas. & 5,45 & 1,954 & 4 & 6 & 7 \\
\hline 3) Todas vacinas do calendário básico. & 5,95 & 4,003 & 2 & 8 & 10 \\
\hline 4) Material impresso para ações em saúde. & 5,53 & 2,164 & 4 & 6 & 7 \\
\hline 7) Medicamentos básicos com regularidade. & 3,68 & 3,146 & 0 & 4 & 6 \\
\hline 8) Mecanismos de monitoramento e controle de estoque e conservação adequada. & 6,11 & 2,628 & 5 & 6 & 8 \\
\hline
\end{tabular}

A média de pontuação total das equipes localizadas na zona rural $(39,82 \pm 4,976)$ não foi diferente, estatisticamente, daquelas situadas na área urbana $(39,11 \pm 14,331)$ : $p=0,872$. Também não foi encontrada diferença estatisticamente significante entre as médias obtidas em cada um dos diferentes oito padrões de qualidade, conforme localização da UBS ( $p>0,05)$ (Tabela 2). 
Tabela 2. Médias de pontos das equipes em cada padrão de qualidade para Insumos, Imunobiológicos e Medicamentos na Unidade Básica de Saúde (UBS) conforme localização urbana ou rural. Equipes de saúde da família, Montes Claros, Minas Gerais, Brasil, 2014. $n=75$

\begin{tabular}{|c|c|c|c|c|c|c|}
\hline Padrão de qualidade (UBS dispõe de...) & \multicolumn{2}{|c|}{ Localização } & $\begin{array}{l}\text { Diferença } \\
\text { média }\end{array}$ & \multicolumn{2}{|c|}{ IC $95 \% *$} & $\mathbf{p}^{* \star * *}$ \\
\hline 1) Insumos para ações de saúde e atividades educativas. & 5,48 & 5,27 & 0,212 & $-1,067$ & 1,491 & 0,742 \\
\hline 3) Todas vacinas do calendário básico. & 5,83 & 6,64 & $-0,808$ & $-3,423$ & 1,807 & 0,426 \\
\hline 4) Material impresso para ações em saúde. & 5,52 & 5,64 & $-0,121$ & $-1,538$ & 1,297 & 0,811 \\
\hline 6) Equipamentos de proteção individual regular e suficiente. & 5,59 & 5,09 & 0,503 & $-0,986$ & 1,992 & 0,503 \\
\hline 7) Medicamentos básicos com regularidade. & 3,52 & 4,64 & $-1,121$ & $-3,164$ & 0,923 & 0,135 \\
\hline $\begin{array}{l}\text { 8) Mecanismos de monitoramento e controle de estoque e } \\
\text { conservação adequada. }\end{array}$ & 6,22 & 5,45 & 0,764 & $-0,948$ & 2,476 & 0,181 \\
\hline
\end{tabular}

A classificação de qualidade quanto aos "Insumos, Imunobiológicos e Medicamentos" foi regular para $34(45,3 \%)$ das UBS e insatisfatório para 22 (29,3\%), segundo avaliação das equipes de saúde da família (Tabela 3).

Tabela 3. Classificação da qualidade das equipes para Insumos, Imunobiológicos e Medicamentos na Unidade Básica de Saúde (UBS). Equipes de saúde da família, Montes Claros, Minas Gerais, Brasil, 2014. n=75

\begin{tabular}{lcc}
\hline & Insumos, Imunobiológicos e Medicamentos & \\
\hline Classificação de qualidade & $\mathbf{n}$ & $\%$ \\
\hline Muito insatisfatório & 2 & 2,7 \\
Insatisfatório & 22 & 29,3 \\
Regular & 34 & 45,3 \\
Satisfatório & 14 & 18,7 \\
Muito satisfatório & 3 & 4,0 \\
Total & 75 & 100,00 \\
\hline
\end{tabular}

A maior parte das unidades da área urbana foi classificada nos níveis insatisfatório e regular; enquanto que, das 11 rurais, 10 ficaram no nível regular. As categorias de qualidade não foram estatisticamente associadas à localização urbana ou rural $(p=0,479)$. Os dois extremos de classificação, muito insatisfatório e muito satisfatório, foram constatados apenas para as UBS da zona urbana (Tabela 4).

\section{Discussão}

\section{Resumo dos principais achados do estudo}

Ao analisar a subdimensão insumos, imunobiológicos e medicamentos da $A M A Q-A B$, este estudo evidenciou uma avaliação, predominantemente, negativa pelos profissionais de saúde da família vinculados ao município investigado, e baixas médias nos padrões de qualidade mensurados. Os itens relativos às 
Tabela 4. Classificação da qualidade das equipes para Insumos, Imunobiológicos e Medicamentos na Unidade Básica de Saúde (UBS) conforme localização urbana ou rural. Equipes de saúde da família, Montes Claros, Minas Gerais, Brasil, 2014. $n=75$

\begin{tabular}{|c|c|c|c|}
\hline \multicolumn{4}{|c|}{ Insumos, Imunobiológicos e Medicamentos } \\
\hline \multirow{2}{*}{ Classificação de qualidade } & \multicolumn{2}{|c|}{ Localização } & \multirow{2}{*}{$\mathbf{p}^{*}$} \\
\hline & Urbana $\mathrm{n}(\%)$ & Rural n (\%) & \\
\hline Muito Insatisfatório & $2(100,0)$ & $0(0,0)$ & \multirow{5}{*}{0,479} \\
\hline Insatisfatório & $22(100,0)$ & $0(0,0)$ & \\
\hline Regular & $24(70,6)$ & $10(29,4)$ & \\
\hline Satisfatório & $13(92,9)$ & $1(7,1)$ & \\
\hline Muito satisfatório & $3(100,0)$ & $0(0,0)$ & \\
\hline
\end{tabular}

* Realizado Teste Qui-quadrado de tendência linear, Linear-by-linear association.

vacinas, à abordagem inicial nas situações de urgência e emergência e à disponibilidade dos medicamentos estiveram como os mais afetados. Sobressaíram-se as classificações de qualidade das equipes em "muito insatisfatória", "insatisfatória" e "regular". Na zona rural somente uma unidade classificou-se como "satisfatória", todas as outras foram classificadas em padrão de qualidade "regular".

\section{Fortalezas e limitações do estudo}

Considera-se o atual estudo de importância fundamental para a avaliação das UBS, quanto à disposição de insumos, imunobiológicos e medicamentos, uma vez que se adotou instrumento de avaliação proposto pelo Ministério da Saúde do Brasil. Todos os itens/padrão de qualidade foram pontuados a partir do consenso entre os profissionais inseridos na saúde da família e, portanto, os mais habilitados a avaliar as condições de trabalho no contexto em que estão inseridos. Também tem como fortaleza o fato de ter agregado novas informações relevantes à literatura nacional sobre a avaliação dos serviços de AB e ESF. Essas informações podem ser aplicadas no cotidiano das equipes e de seus gestores, na autogestão e em busca de melhorias nas UBS.

Entretanto, este trabalho apresenta certas limitações; por caracterizar-se como pesquisa de delineamento transversal, não há como estabelecer a relação de causalidade nas associações detectadas. A abrangência foi restrita à ESF e aos profissionais de saúde da família de um único município, o que dificulta extrapolações para outros cenários. Os dados foram oriundos de autoavaliação das equipes, então, poderiam estar propensos a parâmetros subjetivos e a um possível viés de informação. O tamanho amostral não foi dimensionado para testar hipóteses com um poder estatístico pré-estabelecido, no entanto, o maior número de equipes da ESF participaram do estudo.

\section{Comparação com a literatura já existente}

O presente estudo permitiu evidenciar a autoavaliação dos profissionais que integram as equipes da ESF, quanto aos insumos, imunobiológicos e medicamentos nas UBS. Predominantemente, os itens componentes da subdimensão se enquadraram em baixos padrões de qualidade, com destaque para a classificação regular e insatisfatória. Em publicações sobre a temática qualidade dos serviços primários de saúde, também são identificados achados semelhantes. . $^{8,10,13}$ 
Os resultados desta pesquisa seguem uma tendência nacional: em uma análise da estrutura de 38.812 UBS brasileiras, conforme resultados do PMAQ-AB, verificou-se que a subdimensão instalações e insumos apresentou baixa pontuação média, sendo constatada deficiência de equipamentos, como também de insumos nas unidades. ${ }^{8}$ Em outra investigação, realizada em Belo Horizonte - MG, a oferta de insumos esteve associada à melhor qualidade da assistência na $A B .{ }^{13}$

Tais achados confluem a uma limitação que existe no sistema de saúde nacional, ainda configurado por debilidades e necessidade de mais investimentos na estrutura dos serviços da $A B$. Delineiam uma realidade que limita as ações e a resolutividade dos problemas apresentados nas comunidades da ESF. ${ }^{8}$ As inadequações nas UBS prejudicam a qualidade do cuidado, causam insatisfação para os usuários e os profissionais, além de proporcionarem condições inapropriadas e insalubres de trabalho para as equipes.

Por conseguinte, limitam o potencial dos serviços para a consolidação da ESF, na perspectiva de reorganizar as práticas centradas na tríade indivíduo-família-comunidade e na qualidade da atenção à saúde. ${ }^{10} \mathrm{O}$ enfrentamento da carência estrutural é essencial na oferta de assistência com qualidade, com vistas ao fortalecimento e consolidação do SUS como sistema público universal. ${ }^{8}$

Um importante aspecto presente na autoavaliação das equipes trata-se da disposição da totalidade de vacinas requeridas no calendário básico proposto pelo Programa Nacional e Estadual de Imunização. Nesse quesito, houve expressiva quantidade de equipes com pontuação zero, que representa total inadequação da UBS. No que se refere aos mecanismos para monitoramento e controle de estoque e conservação adequada, a pontuação entre as equipes variou, embora a média geral não tenha sido satisfatória. No cenário local, tem-se como possível explicação o fato de que apenas determinadas UBS são equipadas com sala de vacina e oferecem vacinação à população, logo, nem todas as unidades prestam esse serviço regularmente.

Em pesquisa de base nacional, apenas 7,9\% das equipes atenderam plenamente ao item "ter sempre disponível os imunobiológicos do PMAQ-AB" ${ }^{14}$ Em outro trabalho similar, $82 \%$ das equipes declararam dispor das vacinas previstas no calendário básico. ${ }^{6}$ Sobre disposição de geladeira exclusiva para armazenar as vacinas, no estado do Espírito Santo - Brasil, essa realidade foi constatada para $52 \%$ das unidades. ${ }^{15} \mathrm{Na}$ avaliação da organização e do funcionamento do espaço físico destinado à vacinação, em um município de grande porte de Minas Gerais (MG), registrou-se a necessidade de melhorias estruturais para respeitar as normas instituídas pelo Programa Nacional de Imunização (PNI). ${ }^{16}$

No âmbito do SUS, os municípios devem disponibilizar espaços adequados para a conservar e administrar as vacinas, além de ofertar profissionais devidamente capacitados para gerenciar, conservar e manipular adequadamente os imunobiológicos. Ainda, monitorar e avaliar as ações de imunização nas unidades da ESF. ${ }^{16}$ Todavia, há dificuldades em atender criteriosamente às normas e orientações do PNI, devido principalmente a falhas gerenciais e estruturais no contexto municipal.

$\mathrm{Na}$ atual pesquisa, também se averiguou a existência de insumos e medicamentos recomendados para o primeiro atendimento, tanto para os casos de urgência como para emergência. Esse item obteve a maior frequência de equipes na condição de total inadequação e correspondeu à menor média entre todos os itens analisados. Essa avaliação negativa foi igualmente constatada em outros estudos, conduzidos na macrorregião nordeste ${ }^{17}$ e no norte do estado de MG. ${ }^{10}$ Para esse achado, atribuem-se as hipóteses 
de baixo investimento destinado à aquisição de insumos e medicamentos específicos para situações de urgência e emergência na $A B$; como também o caráter histórico de tais atendimentos, comumente executados apenas em hospitais e ambulatórios especializados. ${ }^{17}$

Portanto, é necessário intervir nesse aspecto, a fim de viabilizar a maior resolubilidade da ESF. ${ }^{17}$ É válido ressaltar que, como a ESF constitui a porta de entrada na rede dos serviços de saúde pública, suas unidades devem estar adequadamente equipadas para uma primeira abordagem, nos casos de urgência e emergência. ${ }^{10}$

Nesta pesquisa, registrou-se uma baixa média de pontuação das equipes para suficiência e regularidade na disponibilidade de medicamentos do Componente Básico da Assistência Farmacêutica, bem como sobre o monitoramento e controle de estoque e conservação desses medicamentos. Essa insuficiência de medicamentos na $A B$ foi igualmente identificada em outras pesquisas avaliativas, ${ }^{13,18-20}$ com igual situação no que concerne às condições de armazenamento e conservação. ${ }^{21,22}$

Tais resultados demonstram uma realidade comprometedora da assistência na ESF, o que suscita a discussão sobre o controle de conservação dos medicamentos, necessária para resguardar a eficiência e segurança dos mesmos. ${ }^{23}$ Os medicamentos apresentam um notável impacto sobre a terapêutica de morbidades, epidemiologicamente relevantes no Brasil, em decorrência da carga de doenças vigente e das implicações do envelhecimento da população. Como exemplo, destacam-se as doenças crônico-degenerativas - sobretudo hipertensão arterial sistêmica e diabetes mellitus. ${ }^{20}$

Assim, as deficiências na disponibilidade de medicamentos comprometem o controle do problema de saúde e a adesão ao tratamento, com agravamento da qualidade de vida do usuário. Fatos que expandem as despesas com assistência, ambulatoriais e internações. Adicionalmente, a maioria dos atendidos nos serviços de saúde da família é de baixa renda, com necessidade de acesso gratuito aos medicamentos, para não prejudicar a integralidade do cuidado. ${ }^{19,20} \mathrm{O}$ acesso a medicamentos essenciais foi considerado, em outros estudos, baixo e irregular no mundo, ${ }^{20,21}$ inclusive no Brasil. ${ }^{21}$

Esse contexto sugere deficiências estruturais e na gestão, no nível municipal, que assegure resolubilidade às demandas de medicamentos. ${ }^{20}$ Após descentralizar a assistência farmacêutica na $A B$, os municípios se responsabilizam pelas etapas de seleção à dispensação dos medicamentos na Atenção Primária. Contudo, são etapas para as quais se requer apoio técnico e financeiro dos governos estaduais e federal, em especial quanto aos repasses de recursos financeiros. Ainda, no caso dos municípios de médio e grande porte, eles enfrentam distintos problemas, devido ao porte populacional e à complexidade do perfil da população e da rede de atenção à saúde,${ }^{18-20}$ quando comparados aos municípios de pequeno porte populacional.

Quanto à análise das categorias de qualidade da subdimensão segundo a localização urbana e rural, não houve resultado estatisticamente significante. Constatou-se somente para as unidades da zona urbana os padrões "muito insatisfatório" e "muito satisfatório". Entretanto, essas UBS, predominantemente, situaram-se nos níveis "insatisfatório" e "regular". Por sua vez, as unidades rurais se concentraram na classificação "regular", nenhuma obteve classificação "muito satisfatória”. Assim, ambas as localizações apresentaram situação negativa, embora se esperasse melhor avaliação na área urbana, com suposição de melhor estruturação dos serviços. 
Esses resultados representam as desigualdades nos arranjos estruturais das UBS, tanto entre as urbanas quanto entre estas e as rurais. Em linhas gerais, tais achados se comportam dentro de uma tendência nacional, posto que investigações prévias revelam precariedades na rede física de unidades de saúde, em termos de infraestrutura, equipamentos, insumos, imunobiológicos e medicamentos. 8,10,20-22 Todavia, é oportuno salientar que o acesso à $A B$ e à rede de atenção à saúde, a oferta e a utilização de serviços de saúde da família têm sido um desafio na atenção à saúde dos usuários de áreas rurais, ${ }^{24-26}$ que possuem singularidades e demandas próprias. ${ }^{25,26}$

As diferenças encontradas podem sinalizar diversidades no processo de institucionalização da ESF, em razão da capacidade de o município gerenciar a $A B,{ }^{6}$ conforme as necessidades dos usuários, reduzindo a desigualdade social e regional, ${ }^{24-26} \mathrm{com}$ qualidade da atenção, seja na zona urbana ou na rural. Nesse intento, é pertinente recomendar ações de maior apoio e suporte especialmente aos serviços e equipes com classificação inadequada.

Por fim, os resultados da autoavaliação das equipes da ESF, desta pesquisa, realçam a necessidade de mais ações direcionadas à qualificação do cuidado.

\section{Implicações para a pesquisa na área e/ou para a prática dos profissionais}

Em síntese, condições favoráveis de insumos, imunobiológicos e medicamentos implicam em melhores atributos para se ofertar adequada assistência à comunidade adscrita. Ademais, fortalecem a possibilidade de utilização dos serviços, colaborando para a consolidação da $A B$, como porta de entrada acolhedora e resolutiva. ${ }^{2,14,27}$ Assim, torna-se possível concretizar os atributos conceituais de um cuidado ampliado, integrador das ações direcionadas à promoção da saúde, prevenção de doenças e reabilitação. ${ }^{5,27}$

Os achados observados sinalizam repercussões para a gestão da $A B$ local, considerando principalmente que, nesta pesquisa, foram incluídas equipes da ESF do município, de médio porte populacional, com adesão ao PMAQ-AB. Também suscitam a necessidade de autoavaliação contínua pelas equipes de saúde da família, bem como novas pesquisas sobre a temática, a fim de aprofundar as evidências científicas e contribuir na implementação de uma cultura de avaliação na $A B$.

\section{Conclusão}

Este estudo apresentou baixas médias nos padrões de qualidade mensurados na subdimensão "Insumos, Imunobiológicos e Medicamentos", no âmbito das unidades de saúde da família. Especial atenção deve ser dada aos seguintes itens analisados: vacinação, primeira abordagem nas situações de urgência e emergência, e disponibilidade dos medicamentos. As unidades cuja classificação revelou situação inadequada requerem um olhar mais atento.

A situação verificada prejudica o elenco de ações e a resolutividade da ESF, pois compromete a atenção integralizada à saúde da comunidade. Diante dos resultados, considera-se necessário reverter as fragilidades averiguadas, no cenário desta pesquisa, a partir do aprimoramento dos serviços da $A B$ enquanto eixo estruturante do SUS, a fim de possibilitar não somente a assistência com qualidade e continuada, mas, também, a integralidade do cuidado. 


\section{Contribuições dos autores}

Lima CA e Moreira KS participaram da concepção do estudo, do planejamento e da coleta de dados, da interpretação dos dados, da redação e revisão do artigo. Abreu MHNG participou da concepção do estudo, da análise e interpretação dos dados e da revisão crítica do artigo. Vieira DMA e Mangueira SAL participaram da concepção do estudo, da coleta de dados, da elaboração do banco de dados e contribuíram na redação do artigo. Vieira MA colaborou na orientação, participou da concepção do estudo, do planejamento da coleta de dados, da interpretação dos dados e da revisão crítica do artigo. Costa SM orientou todas as etapas do estudo e da elaboração do artigo, participou da concepção do estudo, do planejamento da coleta de dados, da análise e interpretação dos dados, da redação e revisão crítica do artigo. Todos os autores leram e aprovaram a versão final do artigo.

\section{Conflitos de interesses}

Os autores declaram a inexistência de conflitos de interesses.

\section{Agradecimentos}

À Secretaria Municipal de Saúde de Montes Claros - MG, pela cessão de dados.

\section{Referências}

1. Centro Brasileiro de Estudos de Saúde (CEBES). Presentation. Saúde Debate. 2014;38(n. esp.):9-10. https://doi.org/10.5935/01031104.2014S001

2. Santos AM, Nóbrega IKS, Assis MMA, Rego de Jesus S, Kochergin CN, Bispo Júnior JP, et al. Desafios à gestão do trabalho e educação permanente em saúde para a produção do cuidado na estratégia saúde da família. Rev APS. 2015;18(1):39-49.

3. Hone T, Rasella D, Barreto ML, Majeed A, Millett C. Association between expansion of primary healthcare and racial inequalities in mortality amenable to primary care in Brazil: a national longitudinal analysis. PLoS Med. 2017;14(5):e1002306. https://doi.org/10.1371/ journal.pmed.1002306

4. Brasil. Ministério da Saúde. Secretaria de Atenção a Saúde. Departamento de Atenção Básica. Autoavaliação para Melhoria do Acesso e da Qualidade - AMAQ. Material de apoio a autoavaliação para as equipes de Atenção Básica (Saúde da Família, AB Parametrizada e Saúde Bucal). Brasília (DF): Ministério da Saúde; 2013.

5. Teixeira MB, Casanova A, Oliveira CCM, Ensgtrom EM, Bodstein RCA. Avaliação das práticas de promoção da saúde: um olhar das equipes participantes do Programa Nacional de Melhoria do Acesso e da Qualidade da Atenção Básica. Saúde Debate. 2014;38(n. esp.):52-68. https://doi.org/10.5935/0103-1104.2014S005

6. Fausto MCR, Giovanella L, Mendonça MHM, Seidl H, Gagno J. A posição da Estratégia Saúde da Família na rede de atenção à saúde na perspectiva das equipes e usuários participantes do PMAQ-AB. Saúde Debate. 2014;38(n. esp.):13-33. https://doi.org/10.5935/01031104.2014S003

7. Rasella D, Harhay MO, Pamponet ML, Aquino R, Barreto ML. Impact of primary health care on mortality from heart and cerebrovascular diseases in Brazil: a nationwide analysis of longitudinal data. BMJ. 2014;349:g4014. https://doi.org/10.1136/bmj.g4014

8. Bousquat A, Giovanella L, Fausto MCR, Fusaro ER, Mendonça MHM, Gagno J, et al. Tipologia da estrutura das unidades básicas de saúde brasileiras: os 5 R. Cad Saúde Pública. 2017;33(8):e00037316. https://doi.org/10.1590/0102-311x00037316

9. Scholz S, Ngoli B, Flessa S. Rapid assessment of infrastructure of primary health care facilities - a relevant instrument for health care systems management. BMC Health Serv Res. 2015;15:183. DOI: http://dx.doi.org/1186/s12913-015-0838-8

10. Moreira KS, Lima CA, Vieira MA, Costa SM. Assessment of infrastructure of family health units and equipment used in primary care actions. Cogitare Enferm. 2017;22(2):e51283. DOI: http://dx.doi.org/10.5380/ce.v22i1.51283 
11. Instituto Brasileiro de Geografia e Estatística (IBGE). Cidades@. Brasil. Minas Gerais. Montes Claros. [Internet] Rio de Janeiro: IBGE; 2018 [acesso 2018 Fev 22]. Disponível em: https://cidades.ibge.gov.br/brasil/mg/montes-claros/panorama

12. Silvério JB. Residência Multiprofissional em Saúde da Família: o caso do Município de Montes Claros. In: Brasil. Ministério da Saúde. Secretaria de Gestão do Trabalho e da Educação na Saúde. Departamento de Gestão da Educação na Saúde. Residência multiprofissional em saúde: experiências, avanços e desafios. Brasília (DF): Ministério da Saúde; 2006.

13. Turci MA, Lima-Costa MF, Macinko J. Influência de fatores estruturais e organizacionais no desempenho da atenção primária à saúde em Belo Horizonte, Minas Gerais, Brasil, na avaliação de gestores e enfermeiros. Cad Saúde Pública. 2015;31(9):1941-52. https://doi. org/10.1590/0102-311X00132114

14. Alves MGM, Casotti E, Oliveira LGD, Machado MTC, Almeida PF, Corvino MPF, et al. Fatores condicionantes para o acesso às equipes da Estratégia Saúde da Família no Brasil. Saúde Debate. 2014;38(n. esp.):34-51. https://doi.org/10.5935/0103-1104.2014S004

15. Garcia ACP, Andrade MAC, Zandonade E, Prado TN, Freitas PSS, Cola JP, et al. Análise da organização da Atenção Básica no Espírito Santo: (des)velando cenários. Saúde Debate. 2014;38(n. esp.):221-36. DOI: http://dx.doi.org/10.5935/0103-1104.2014S017

16. Siqueira LDG, Martins AMEBL, Versiani CMC, Almeida LAV, Oliveira CS, Nascimento JE, et al. Assessment of the organization and operation of vaccine rooms in primary health care in Montes Claros, Minas Gerais, Brazil, 2015. Epidemiol Serv Saúde. 2017;26(3):55768. https://doi.org/10.5123/S1679-49742017000300013

17. Cardoso AVL, Chain APN, Mendes RIP, Ferreira e Ferreira E, Vargas AMD, Martins AMEBL, et al. Assessment of the management of the Family Health Strategy via the tool Assessment for Quality Improvement in municipalities of Minas Gerais, Brazil. Ciênc Saúde Coletiva. 2015;20(4):1267-84. https://doi.org/10.1590/1413-81232015204.01832014

18. Mendes LV, Campos MR, Chaves GC, Silva RM, Freitas PS, Costa KS, et al. Disponibilidade de medicamentos nas unidades básicas de saúde e fatores relacionados: uma abordagem transversal. Saúde Debate. 2014;38(n. esp.):109-23. https://doi.org/10.5935/01031104.2014S009

19. Carvalho Filha FSS, Nogueira LT, Medina MG. Avaliação do controle de hipertensão e diabetes na Atenção Básica: perspectiva de profissionais e usuários. Saúde Debate. 2014;38(n. esp.):265-78. https://doi.org/10.5935/0103-1104.2014S020

20. Nascimento RCRM, Álvares J, Guerra Junior AA, Gomes IC, Costa EA, Leite SN, et al. Availability of essential medicines in primary health care of the Brazilian Unified Health System. Rev Saúde Pública. 2017;51(Suppl. 2):10s. https://doi.org/10.11606/S15188787.2017051007062

21. Leite SN, Manzini F, Álvares J, Guerra Junior AA, Costa EA, Acurcio FA, et al. Infrastructure of pharmacies of the primary health care in the Brazilian Unified Health System: analysis of PNAUM - services data. Rev Saúde Pública. 2017;51(Suppl. 2):13s. https://doi.org/10.11606/ S1518-8787.2017051007120

22. Costa EA, Araújo PS, Pereira MT, Souto AC, Souza GS, Guerra Junior AA, et al. Technical issues and conservation conditions of medicines in the primary health care of the Brazilian Unified Health System. Rev Saúde Pública. 2017;51(Suppl. 2):12s. https://doi.org/10.11606/ S1518-8787.2017051007106

23. Barata-Silva C, Hauser-Davis RA, Silva ALO, Moreira JC. Desafios ao controle da qualidade de medicamentos no Brasil. Cad Saúde Coletjva. 2017;25(3):362-70. https://doi.org/10.1590/1414-462x201700030075

24. Moreira KS, Vieira MA, Costa SM. Qualidade da Atenção Básica: avaliação das Equipes de Saúde da Família. Saúde Debate. 2016;40(111):117-27. https://doi.org/10.1590/0103-1104201611109

25. Garnelo L, Lima JG, Rocha ESC, Herkrath FJ. Access and coverage of Primary Health Care for rural and urban populations in the northern region of Brazil. Saúde Debate. 2018;42(n esp. 1):81-99. https://doi.org/10.1590/0103-11042018s106

26. Gage AD, Leslie HH, Bitton A, Jerome JG, Thermidor R, Joseph JP, et al. Assessing the quality of primary care in Haiti. Bull World Health Organ. 2017;95(3):182-90. https://doi.org/10.2471/BLT.16.179846

27. Chueiri PS, Harzheim E, Takeda SMP. Coordenação do cuidado e ordenação nas redes de atenção pela Atenção Primária à Saúde - uma proposta de itens para avaliação destes atributos. Rev Bras Med Fam Comunidade.2017;12(39):1-18. https://doi.org/10.5712/ rbmfc12(39)1363 